\title{
Deux humanistes parlent de leur mort à venir
}

\section{Jean Martin}

Dr méd., membre de la rédaction

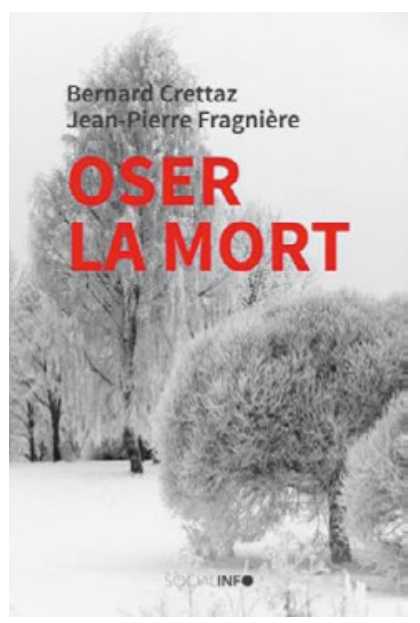

Bernard Crettaz, Jean-Pierre Fragnière Oser la mort

Lausanne: Editions Socialinfo; 2017. 164 pages. $26 \mathrm{CHF}$.

Ces deux auteurs sont bien connus en Suisse romande. Bernard Crettaz, ancien conservateur au Musée d'ethnographie de Genève, a créé le concept de «Cafés mortels» où on échange informellement sur la mort, dont il a animé une centaine de réunions (concept repris dans plusieurs pays - voir pages 59 à 64). Jean-Pierre Fragnière, qui a enseigné la sociologie à l'Ecole d'études sociales et pédagogiques de Lausanne et à l'Université de Genève, a eu une intense activité d'éditeur depuis plus de trente ans. Les deux sont originaires du Valais, dans un milieu rural au cadre catholique strict.

A la retraite mais très actifs, ils écrivent sur la mort. Crettaz enrichi par son expérience des Cafés mortels.

\section{A la retraite mais très actifs, ils écrivent sur la mort.}

Fragnière lui notamment sur la base de son expérience souvent lourde, depuis plusieurs années, au contact de la médecine et des hôpitaux (traitements anti-cancé- reux, transplantation). Tout académiques qu'ils sont, ils sont ainsi des explorateurs de la mort sur des plans très pratiques.

\section{La société ancienne et la nouvelle}

«Nous avons été élevés entre le catéchisme, le régent voire le gendarme. Tout cela sous l'œil vigilant de la voisine occupée à assumer le contrôle social, l'efficace ancêtre de nos caméras modernes. On apprenait très tôt qui était habilité à définir les règles et à trancher les conflits. La rapide fragilisation de ce système a ouvert des espaces au marché de la gestion des comportements et des prestations de soutien à la vie privée. Des cohortes de professionnels ont offert leurs services [...]

\section{Crettaz se décrit comme vivant le vieillisse- ment "par glissade», descente de la pente par le poids des ans.}

On observe une véritable marchandisation de la mort. Parmi d'autres les croque-morts en sont les éminents représentants. Ils ont pris la place des clergés.» Citation de Emile Durkheim: "Comment se fait-il que, tout en devenant plus autonome, l'individu dépende plus étroitement de la société?» Plus un système devient complexe, plus il pourra développer son autonomie, plus il aura des dépendances multiples - réflexion aussi développée par Edgar Morin.

\section{Deux types de cheminements}

Crettaz se décrit comme vivant le vieillissement "par glissade», descente de la pente par le poids des ans: «Je me trouve très seul à l'heure où les faits me signifient que je suis vieux, lorsque des tremblements grippent les gestes quotidiens, quand la sauce tache ma chemise, lorsque je me mets à jauger un appartement en fonction de l'accessibilité des toilettes.» Fragnière lui, parlant de lui et d'autres comme de survivants (à la faveur des progrès médicaux), décrit une «vie et mort en escalier», avec la répétition qu'il a connue de soucis graves 
et de retours à meilleure santé - un "cache-cache avec la mort», avec des sursis longs. "Qui n'a pas un survivant dans son entourage? Il faut prendre la mesure des conséquences de ces succès que nous avons construits et mis en œuvre. Mais aussi, chacun sait que dans l'escalier il existe la dernière marche.»

\section{Vers la fin...}

«Sur les rivages de la fin, nous ressentons une forte invitation à desserrer les liens, à faire le vide, à laisser place aux interrogations. Nous devinons qu'il sera impossible d'échapper à l'hésitation et aux incertitudes.» Evocation de l'option de décider de s'en aller quand la vie devient trop lourde (éventualité du suicide assisté) aux pages 107 et 108. «Pourtant, ils reviennent à la charge, tous ceux qui veulent nous inviter à souffrir en silence, à vivre notre déchéance jusqu'au bout. Ne pas succomber à la tentation de mettre fin au calvaire. Avec quel argument: il ne faut pas faire de peine à ceux qui restent. A méditer.» «Lorsqu'elle survient, la mort déclenche une avalanche de scénarios construits comme des comédies ou des passages obligés, qu'il faudrait dénoncer comme des hypocrisies. Mais il est bon, aussi, de reconnaître le côté bénéfique de ce théâtre. Il permet un acte fondamental: gérer un moment de passage, tous les rites obéissent à une dramaturgie.»

\section{Conclusion}

"Au terme de l'aventure de ce livre, nous savons ce que nous devons aux vivants, nous reconnaissons également tout ce que nous devons aux morts. A tous ceux qui ont arrosé la société dans laquelle nous avons trouvé notre chemin, qui vivent en nous parce qu'ils nous ont transmis leur patrimoine et guidé beaucoup de nos choix. Et aussi parce qu'ils nous ont laissé vivre.»

Pour chacun, ce livre apporte du grain à moudre, il fait se demander si on ne devrait pas consacrer plus d'at-

\section{Pour chacun, ce livre apporte du grain à} moudre, il fait se demander si on ne devrait pas consacrer plus d'attention à la mort qui va venir.

tention à la mort qui va venir. Ceci sans alarmisme ni «dirigisme», sur un mode dialoguant, proche de la vie pratique - et de la mort pratique, dans des pages relevant ce qu'il fau(drai)t préparer en vue de sa propre mort ou décrivant, parfois, des démarches funéraires. Pour finir, cette parole de Sénèque: «Personne ne se soucie de bien vivre mais de vivre longtemps, alors que tous peuvent se donner le bonheur de bien vivre, aucun de vivre longtemps.» 